Bull. Austral. Math. Soc.

$47 \mathrm{H} 09,49 \mathrm{M} 20,47 \mathrm{~A} 35$

VOL. $66(2002) \quad[9-16]$

\title{
CONSTRUCTION OF SUNNY NONEXPANSIVE RETRACTIONS IN BANACH SPACES
}

\author{
Tomas Dominguez Benavides, Genaro López Acedo and Hong-Kun Xu
}

\begin{abstract}
Let $\mathcal{J}$ be a commutative family of nonexpansive self-mappings of a closed convex subset $C$ of a uniformly smooth Banach space $X$ such that the set of common fixed points is nonempty. It is shown that if a certain regularity condition is satisfied, then the sunny nonexpansive retraction from $C$ to $F$ can be constructed in an iterative way.
\end{abstract}

\section{INTRODUCTION}

Let $C$ be a nonempty closed convex subset of a Banach space $X$ and let $D$ be a nonempty subset of $C$. A retraction from $C$ to $D$ is a mapping $Q: C \rightarrow D$ such that $Q x=x$ for $x \in D$. A retraction $Q$ from $C$ to $D$ is nonexpansive if $Q$ is nonexpansive (that is, $\|Q x-Q y\| \leqslant\|x-y\|$ for $x, y \in C$ ). A retraction $Q$ from $C$ to $D$ is sunny if $Q$ satisfies the property:

$$
Q(Q x+t(x-Q x))=Q x \quad \text { for } x \in C \text { and } t>0 \text { whenever } Q x+t(x-Q x) \in C \text {. }
$$

A retraction $Q$ from $C$ to $D$ is sunny nonexpansive if $Q$ is both sunny and nonexpansive. It is known ([2]) that in a smooth Banach space $X$, a retraction $Q$ from $C$ to $D$ is a sunny nonexpansive retraction from $C$ to $D$ if and only if the following inequality holds:

$$
\langle x-Q x, J(y-Q x)\rangle \leqslant 0, \quad x \in C, y \in D,
$$

where $J: X \rightarrow X^{*}$ is the duality map defined by

$$
J x:=\left\{x^{*} \in X^{*}:\left\langle x, x^{*}\right\rangle=\|x\|^{2}=\left\|x^{*}\right\|^{2}\right\}, \quad x \in X .
$$

Hence a sunny nonexpansive retraction must be unique (if it exists).

If $C$ is a nonempty closed convex subset of a Hilbert space $H$, then the nearest point projection $P_{C}$ from $H$ to $C$ is the sunny nonexpansive retraction. This however is not true for Banach spaces since nonexpansivity of projections $P_{C}$ characterises Hilbert spaces.

Received 15th November, 2001

The first and second authors were supported in part by project BFM 2000-0344 and FQM-127. The third author was supported in part by NRF (South Africa) and D.G.U. SAB 1998-0116 (Spain).

Copyright Clearance Centre, Inc. Serial-fee code: 0004-9727/02 \$A2.00+0.00. 
On the other hand, one sees by (1.1) that a sunny nonexpansive retraction can play a similar role in a Banach space as a projection does in a Hilbert space. So an interesting problem is this: In what kind of Banach spaces, does a sunny nonexpansive retraction exist? If it does exist, how can one find it? It is known ([2]) if $C$ is a closed convex subset of a uniformly smooth Banach space $X$ and there is a nonexpansive retraction from $X$ to $C$, then there exists a sunny nonexpansive retraction from $X$ to $C$. But Bruck's proof is not constructive.

The purpose of the present paper is to construct sunny nonexpansive retractions in a uniformly smooth Banach space in an iterative way. More precisely, we show that if $F$ is the nonempty common fixed point set of a commutative family of nonexpansive selfmappings of a closed convex subset $C$ of a uniformly smooth Banach space $X$ satisfying certain regularity condition, then we are able to construct a sequence that converges to the sunny nonexpansive retraction $Q$ of $C$ to $F$. This extends a result of Reich [7] where the case of a single nonexpansive mapping is dealt with.

\section{Two Lemmas}

LEMMA 2.1. Let $\left(s_{n}\right)$ be a sequence of nonnegative numbers satisfying the condition:

$$
s_{n+1} \leqslant\left(1-\alpha_{n}\right)\left(s_{n}+\beta_{n}\right)+\alpha_{n} \gamma_{n}, \quad n \geqslant 0,
$$

where $\left(\alpha_{n}\right),\left(\beta_{n}\right),\left(\gamma_{n}\right)$ are sequences of real numbers satisfying

(i) $\left(\alpha_{n}\right) \subset[0,1], \lim _{n} \alpha_{n}=0$, and $\sum_{n=0}^{\infty} \alpha_{n}=\infty$, or equivalently, $\prod_{n=0}^{\infty}\left(1-\alpha_{n}\right)$ $:=\lim _{n \rightarrow \infty} \prod_{k=0}^{n}\left(1-\alpha_{k}\right)=0$;

(ii) $\limsup _{n \rightarrow \infty} \beta_{n} \leqslant 0$;

(iii) $\limsup _{n \rightarrow \infty} \gamma_{n} \leqslant 0$.

Then $\lim _{n \rightarrow \infty} s_{n}=0$.

Proof: For any $\varepsilon>0$, let $N \geqslant 1$ be an integer big enough so that

$$
\beta_{n}<\varepsilon / 2, \quad \gamma_{n}<\varepsilon, \quad \alpha_{n}<1 / 2, \quad n \geqslant N .
$$

It follows from (2.1) and (2.2) that, for $n>N$,

$$
\begin{aligned}
s_{n+1} & \leqslant\left(1-\alpha_{n}\right)\left(s_{n}+\beta_{n}\right)+\varepsilon \alpha_{n} \\
& \leqslant\left(1-\alpha_{n}\right)\left(1-\alpha_{n-1}\right)\left(s_{n-1}+\beta_{n-1}\right)+\varepsilon\left(1-\left(1-\alpha_{n}\right)\left(1-\alpha_{n-1}\right)+\frac{1}{2}\left(1-\alpha_{n}\right)\right) \\
& \leqslant\left(1-\alpha_{n}\right)\left(1-\alpha_{n-1}\right)\left(s_{n-1}+\beta_{n-1}\right)+\varepsilon\left(1-\left(\frac{1}{2}-\alpha_{n}\right)\left(\frac{1}{2}-\alpha_{n-1}\right)\right) .
\end{aligned}
$$


Hence by induction we obtain

$$
s_{n+1} \leqslant \prod_{N}^{n}\left(1-\alpha_{j}\right)\left(s_{N}+\beta_{N}\right)+\varepsilon\left[1-\prod_{j=N}^{n}\left(1-\tilde{a}_{j}\right)\right], \quad n>N,
$$

where $\tilde{\alpha}_{j}:=1 / 2+\alpha_{j}<1, j \geqslant N$. By condition (i) we deduce, after taking the limsup as $n \rightarrow \infty$ in the last inequality, that $\limsup s_{n+1} \leqslant \varepsilon$.

Lemma 2.2. (The Subdifferential Inequality, see [3].) In a Banach space $X$ there holds the inequality:

$$
\|x+y\|^{2} \leqslant\|x\|^{2}+2\langle y, j\rangle, \quad x, y \in X, j \in J(x+y),
$$

where $J$ is the duality map of $X$.

\section{Iterative Processes}

Let $G$ be an unbounded subset of $\mathrm{R}^{+}$such that $s+t \in G$ whenever $s, t \in G$. (Often $G=\mathcal{N}$, the set of nonnegative integers or $\mathbf{R}^{+}$.) Let $X$ be a uniformly smooth Banach space, $C$ a nonempty closed convex subset of $X$, and $\mathcal{J}=\left\{T_{s}: s \in G\right\}$ a commutative family of nonexpansive self-mappings of $C$. Denote by $F$ the set of common fixed point of $\mathcal{J}$, that is, $F=\left\{x \in C: T_{s} x=x, s \in G\right\}$. Throughout this section we always assume that $F$ is nonempty. Our purpose is to construct the sunny nonexpansive retraction $Q$ from $C$ to $F$. We shall introduce two iterative processes to construct $Q$. The first one is implicit, while the second one is explicit. But before introducing the iterative processes, we recall that the uniform smoothness of $X$ is equivalent to the following statement:

$$
\lim _{\lambda \rightarrow 0^{+}} \frac{\|x+\lambda y\|^{2}-\|x\|^{2}}{\lambda}=2\langle y, J x\rangle \quad \text { uniformly for bounded } x, y \in X .
$$

Let $u \in C$ be given arbitrarily and let $\left(\alpha_{s}\right)_{s \in G}$ be a net in the interval $(0,1)$ such that $\lim _{s \rightarrow \infty} \alpha_{s}=0$. By Banach's contraction principle, for each $s \in G$, we have a unique point $z_{s} \in C$ satisfying the equation

$$
z_{s}=\alpha_{s} u+\left(1-\alpha_{s}\right) T_{s} z_{s}
$$

Theorem 3.1. Let $X$ be a uniformly smooth Banach space. Assume $\mathcal{J}$ is uniformly asymptotically regular on bounded subsets of $C$; that is, for each bounded subset $\tilde{C}$ of $C$ and each $r \in G$,

$$
\lim _{\substack{s \rightarrow \mathcal{C} \\ s \rightarrow \infty}} \sup _{x \in \tilde{C}}\left\|T_{r} T_{s} x-T_{s} x\right\|=0 .
$$

Then the net $\left(z_{s}\right)$ defined in (3.1) converges in norm and the limit defines the sunny nonexpansive retraction $Q$ from $C$ to $F$. 
Proof: First we observe that $\left(z_{s}\right)$ is bounded. Indeed, for $p \in F$, we have

$$
\left\|z_{s}-p\right\| \leqslant \alpha_{s}\|u-p\|+\left(1-\alpha_{s}\right)\left\|T_{s} z_{s}-p\right\| \leqslant \alpha_{s}\|u-p\|+\left(1-\alpha_{s}\right)\left\|z_{s}-p\right\|
$$

This implies that $\left\|z_{s}-p\right\| \leqslant\|u-p\|$ and $\left(z_{s}\right)$ is bounded. Thus $\left\|z_{s}-T_{s} z_{s}\right\|=\alpha_{s} \| u$ $-T_{s} z_{s} \| \rightarrow 0(s \rightarrow \infty)$. Let $\left(s_{n}\right)$ be a subsequence of $G$ such that $\lim _{n} s_{n}=\infty$. Next we define a function $f$ on $C$ by

$$
f(x):=\operatorname{LIM}_{n}\left\|z_{n}-x\right\|^{2}, \quad x \in C,
$$

where LIM is a Banach limit and $z_{n}:=z_{s_{n}}$. We have for each $r \in G$,

$$
\begin{aligned}
f\left(T_{r} x\right) & =\operatorname{LIM}_{n}\left\|z_{n}-T_{r} x\right\|^{2} \\
& =\operatorname{LIM}_{n}\left\|T_{r} T_{s_{n}} z_{n}-T_{r} x\right\|^{2} \\
& \leqslant \operatorname{LIM}_{n}\left\|T_{s_{n}} z_{n}-x\right\|^{2}
\end{aligned}
$$

using (3.2). Therefore,

$$
f\left(T_{\tau} x\right) \leqslant f(x), \quad r \in G, x \in C
$$

Let

$$
K:=\left\{x \in C: f(x)=\min _{C} f\right\} .
$$

Then it can be seen that $K$ is a closed bounded convex nonempty subset of $C$. By (3.3) we see that $K$ is invariant under each $T_{r}$; that is, $T_{r}(K) \subset K, r \in G$. By Lim [5] the family $\mathcal{J}=\left\{T_{s}: s \in G\right\}$ has a common fixed point, that is, $K \cap F \neq \emptyset$. Let $q \in K \cap F$. Since $q$ is a minimiser of $f$ over $C$ and since $X$ is uniformly smooth, it follows that for each $x \in C$,

$$
\begin{aligned}
0 & \leqslant \lim _{\lambda \rightarrow 0^{+}} \frac{1}{\lambda}[f(q+\lambda(x-q))-f(q)] \\
& =\operatorname{LIM}_{n}\left(\lim _{\lambda \rightarrow 0^{+}} \frac{1}{\lambda}\left[\left\|\left(z_{n}-q\right)+\lambda(q-x)\right\|^{2}-\left\|z_{n}-q\right\|^{2}\right]\right) \\
& =\operatorname{LIM}_{n}\left\langle q-x, J\left(z_{n}-q\right)\right\rangle .
\end{aligned}
$$

Thus,

$$
\operatorname{LIM}_{n}\left\langle x-q, J\left(z_{n}-q\right)\right\rangle \leqslant 0, \quad x \in C
$$

In particular,

$$
\operatorname{LIM}_{n}\left\langle u-q, J\left(z_{n}-q\right)\right\rangle \leqslant 0, \quad x \in C .
$$

On the other hand, by equation (3.1) we have for any $p \in F$,

$$
z_{n}-p=\left(1-\alpha_{n}\right)\left(T_{n} z_{n}-p\right)+\alpha_{n}(u-p)
$$


where $\alpha_{n}=\alpha_{s_{n}}$ and $T_{n}=T_{s_{n}}$. It follows that for $p \in F$,

$$
\begin{aligned}
\left\|z_{n}-p\right\|^{2} & =\left(1-\alpha_{n}\right)\left\langle T_{n} z_{n}-p, J\left(z_{n}-p\right)\right\rangle+\alpha_{n}\left\langle u-p, J\left(z_{n}-p\right)\right\rangle \\
& \leqslant\left(1-\alpha_{n}\right)\left\|z_{n}-p\right\|^{2}+\alpha_{n}\left\langle u-p, J\left(z_{n}-p\right)\right\rangle .
\end{aligned}
$$

Hence

$$
\left\|z_{n}-p\right\|^{2} \leqslant\left\langle u-p, J\left(z_{n}-p\right)\right\rangle .
$$

Combining (3.4) and (3.5) with $p$ replaced with $q$, we get

$$
\operatorname{LIM}_{n}\left\|z_{n}-q\right\|^{2} \leqslant 0 .
$$

So we have a subsequence $\left(z_{n_{j}}\right)$ of $\left(z_{n}\right)$ such that $z_{n_{j}} \stackrel{s}{\longrightarrow} q$. Assume there exists another subsequence $\left(z_{m_{k}}\right)$ of $\left(z_{s}\right)$ such that $z_{m_{k}} \stackrel{s}{\longrightarrow} \widetilde{q}$. Then (3.5) implies

$$
\|q-\widetilde{q}\|^{2} \leqslant\langle u-\widetilde{q}, J(q-\widetilde{q})\rangle .
$$

Similarly we have

$$
\|\tilde{q}-q\|^{2} \leqslant\langle u-q, J(\tilde{q}-q)\rangle .
$$

Adding up (3.6) and (3.7) obtains $q=\tilde{q}$. Therefore $\left(z_{s}\right)$ converges in norm to a point in $F$.

Now define $Q: C \rightarrow F$ by

$$
Q u:=s-\lim _{s \rightarrow \infty} z_{s}
$$

Then $Q$ is a retraction from $C$ to $F$. Moreover, by (3.5) we get for $p \in F$,

$$
\|Q u-p\|^{2} \leqslant\langle u-p, J(Q u-p)\rangle \Rightarrow\langle u-Q u, J(p-Q u)\rangle \leqslant 0, \quad p \in F .
$$

Therefore $Q$ is a sunny nonexpansive retraction from $C$ to $F$.

Next we introduce an explicit iterative process to construct the sunny nonexpansive retraction $Q$ from $C$ to $F$.

Let $u \in C$ be arbitrary. Take a sequence $\left(r_{n}\right)$ in $G$ and a sequence $\left(\alpha_{n}\right)$ in the interval $[0,1]$. Starting with an arbitrary initial $x_{0} \in C$ we define a sequence $\left(x_{n}\right)$ recursively by the formula:

$$
x_{n+1}:=\alpha_{n} u+\left(1-\alpha_{n}\right) T_{r_{n}} x_{n}, \quad n \geqslant 0 .
$$

The following is a convergence result for the process (3.8).

TheOREM 3.2. Let $X$ be a uniformly smooth Banach space. Assume

(i) $\alpha_{n} \rightarrow 0, \alpha_{n} / \alpha_{n+1} \rightarrow 1$, and $\sum_{n} \alpha_{n}=\infty$; 
(ii) . $r_{n} \rightarrow \infty$;

(iii) $\mathcal{J}$ is semigroup (that is, $T_{r} T_{s}=T_{r+s}$ for $r, s \in G$ ) and satisfies the uniformly asymptotically regularity condition:

$$
\lim _{r \in G} \sup _{\substack{r \\ \rightarrow}}\left\|T_{s} T_{r} x-T_{r} x\right\|=0 \quad \text { uniformly in } s \in G
$$

where $\widetilde{C}$ is any bounded subset of $C$. Then the sequence $\left(x_{n}\right)$ generated by (3.8) converges in norm to $Q u$, where $Q$ is the sunny nonexpansive retraction from $C$ to $F$ established in Theorem 3.1.

Proof: 1. First prove the sequence $\left(x_{n}\right)$ is bounded. As a matter of fact, for $p \in F$, we have

$$
\left\|x_{n+1}-p\right\| \leqslant \alpha_{n}\|u-p\|+\left(1-\alpha_{n}\right)\left\|x_{n}-p\right\| .
$$

This together with an induction implies that

$$
\left\|x_{n}-p\right\| \leqslant \max \left\{\|u-p\|,\left\|x_{0}-p\right\|\right\}, \quad \text { for all } n \geqslant 0
$$

Thus $\left(x_{n}\right)$ is bounded and it follows that

$$
\left\|x_{n+1}-T_{r_{n}} x_{n}\right\|=\alpha_{n}\left\|u-T_{r_{n}} x_{n}\right\| \rightarrow 0 .
$$

2. Now prove $\left\|x_{n+1}-x_{n}\right\| \rightarrow 0$. Indeed we have

$$
\begin{aligned}
\left\|x_{n+1}-x_{n}\right\|= & \|\left(\alpha_{n}-\alpha_{n-1}\right)\left(u-T_{r_{n-1}} x_{n-1}\right) \\
& \quad+\left(1-\alpha_{n}\right)\left(T_{r_{n}} x_{n}-T_{r_{n}} x_{n-1}\right)+\left(1-\alpha_{n}\right)\left(T_{r_{n}} x_{n-1}-T_{r_{n-1}} x_{n-1}\right) \| \\
\leqslant & \quad+\alpha_{n}-\alpha_{n-1} \mid\left\|u-T_{r_{n-1}} x_{n-1}\right\|+\left(1-\alpha_{n}\right)\left\|x_{n}-x_{n-1}\right\| \\
& \quad+\left(1-\alpha_{n}\right)\left\|T_{r_{n}} x_{n-1}-T_{r_{n-1}} x_{n-1}\right\| \\
\leqslant & \quad\left(1-\alpha_{n}\right)\left(\left\|x_{n}-x_{n-1}\right\|+\beta_{n}\right)+\alpha_{n} \gamma_{n},
\end{aligned}
$$

where $\beta_{n}:=\left\|T_{r_{n}} x_{n-1}-T_{r_{n-1}} x_{n-1}\right\|$ and $\gamma_{n}:=\left(\alpha_{n}^{-1}\right)\left|\alpha_{n}-\alpha_{n-1}\right|\left\|u-T_{r_{n-1}} x_{n-1}\right\|$. Since $\left(x_{n}\right)$ is bounded, by condition (i), we have $\gamma_{n} \rightarrow 0$. It is easily seen that condition (iii) implies $\beta_{n} \rightarrow 0$. Indeed, if $r_{n}>r_{n-1}$, since $\mathcal{J}$ is a semigroup, we have $\beta_{n}$ $=\left\|T_{r_{n}-r_{n-1}} T_{r_{n-1}} x_{n-1}-T_{r_{n-1}} x_{n-1}\right\| \rightarrow 0$ by step 1 and (3.9). Interchanging $r_{n}$ and $r_{n-1}$ if $r_{n}<r_{n-1}$ finishes the proof of $\beta_{n} \rightarrow 0$. Hence by Lemma 2.1 we get $\left\|x_{n+1}-x_{n}\right\| \rightarrow 0$.

3. Next we show for each fixed $s \in G,\left\|T_{s} x_{n}-x_{n}\right\| \rightarrow 0$. Indeed (3.10) and step 2 imply that $\left\|x_{n}-T_{r_{n}} x_{n}\right\| \rightarrow 0$. Let $\widetilde{C}$ be any bounded subset of $C$ which contains the sequence $\left(x_{n}\right)$. It follows that

$$
\begin{aligned}
\left\|T_{s} x_{n}-x_{n}\right\| & \leqslant\left\|T_{s} x_{n}-T_{s} T_{r_{n}} x_{n}\right\|+\left\|T_{s} T_{r_{n}} x_{n}-T_{r_{n}} x_{n}\right\|+\left\|T_{r_{n}} x_{n}-x_{n}\right\| \\
& \leqslant 2\left\|x_{n}-T_{r_{n}} x_{n}\right\|+\sup _{x \in \tilde{C}}\left\|T_{s} T_{r_{n}} x-T_{r_{n}} x\right\| .
\end{aligned}
$$


So condition (iii) implies that $\left\|T_{s} x_{n}-x_{n}\right\| \rightarrow 0$.

4. Now we prove $\limsup _{n \rightarrow \infty}\left\langle u-q, J\left(x_{n}-q\right)\right\rangle \leqslant 0$, where $q=Q(u)$. Recall that $z_{s}$ satisfies (3.1) and we have shown that $z_{s} \stackrel{s}{\longrightarrow} q$. Writing

$$
z_{s}-x_{n}=\alpha_{s}\left(u-x_{n}\right)+\left(1-\alpha_{s}\right)\left(T_{r_{s}} z_{s}-x_{n}\right)
$$

and applying Lemma 2.2 we get

$$
\begin{aligned}
\left\|z_{s}-x_{n}\right\|^{2} & \leqslant\left(1-\alpha_{s}\right)^{2}\left\|T_{r_{s}} z_{s}-x_{n}\right\|^{2}+2 \alpha_{s}\left\langle u-x_{n}, J\left(z_{s}-x_{n}\right)\right\rangle \\
& \leqslant\left(1+\alpha_{s}^{2}\right)\left\|z_{s}-x_{n}\right\|^{2}+M\left\|x_{n}-T_{r_{s}} x_{n}\right\|+2 \alpha_{s}\left\langle u-z_{s}, J\left(z_{s}-x_{n}\right)\right\rangle,
\end{aligned}
$$

where $M$ is a constant such that $\left\|x_{n}-T_{r_{s}} x_{n}\right\|+2\left\|z_{s}-x_{n}\right\| \leqslant M$ for all $n, s \in G$. It follows from the last inequality that

$$
\left\langle u-z_{s}, J\left(x_{n}-z_{s}\right)\right\rangle \leqslant \frac{\alpha_{s}}{2}\left\|z_{s}-x_{n}\right\|^{2}+\frac{M}{2 \alpha_{s}}\left\|T_{r_{s}} x_{n}-x_{n}\right\| .
$$

By step 3 we find that

$$
\limsup _{n \rightarrow \infty}\left\langle u-z_{s}, J\left(x_{n}-z_{s}\right)\right\rangle \leqslant O\left(\alpha_{s}\right) \rightarrow 0 \text { as } s \rightarrow \infty .
$$

Since $X$ is uniformly smooth, the duality map $J$ is norm-to-norm uniformly continuous on bounded subsets of $X$, letting $s \rightarrow \infty$ in (3.11) we obtain $\limsup _{n \rightarrow \infty}\left\langle u-q, J\left(x_{n}-q\right)\right\rangle \leqslant 0$.

5. Finally we show $x_{n} \rightarrow q$ in norm. Apply Lemma 2.2 to get

$$
\begin{aligned}
\left\|x_{n+1}-q\right\|^{2} & =\left\|\left(1-\alpha_{n}\right)\left(T_{T_{n}} x_{n}-q\right)+\alpha_{n}(u-q)\right\|^{2} \\
& \leqslant\left(1-\alpha_{n}\right)^{2}\left\|T_{r_{n}} x_{n}-q\right\|^{2}+2 \alpha_{n}\left\langle u-q, J\left(x_{n+1}-q\right)\right\rangle \\
& \leqslant\left(1-\alpha_{n}\right)\left\|x_{n}-q\right\|^{2}+2 \alpha_{n}\left\langle u-q . J\left(x_{n+1}-q\right)\right\rangle .
\end{aligned}
$$

Now using, condition (i), step 4 and Lemma $2.1\left(\beta_{n} \equiv 0\right)$ we conclude that $\left\|x_{n}-q\right\| \rightarrow 0$.

REMARKS. 1 . The case where the family $\mathcal{J}$ consists of a single nonexpansive mapping was studied by Reich [7]. For the framework of a Hilbert spaces see also Halpern [4], Lions [6] and Wittmann [8].

2. For the case where $\mathcal{J}$ is a finite family of nonexpansive mappings and $X$ is a Hilbert space, similar results involving with a periodic iteration process were proved in Lions [6] and Bauschke [1].

\section{REFERENCES}

[1] H.H. Bauschke, 'The approximation of fixed points of compositions of nonexpansive mappings in Hilbert spaces', J. Math. Anal. Appl. 202 (1996), 150-159.

[2] R.E. Bruck, 'Nonexpansive projections on subsets of Banach spaces', Pacific J. Math. 47 (1973), 341-355. 
[3] I. Cioranescu, Geometry of Banach spaces, duality mappings and nonlinear problems, Mathematics and its Applications 62 (Kluwer Academic Press, Dordrecht, 1990).

[4] B. Halpern, 'Fixed points of nonexpanding maps', Bull. Amer. Math. Soc. 73 (1967), 957-961.

[5] T.C. Lim, 'A fixed point theorem for families on nonexpansive mappings', Pacific $J$. Math. 53 (1974), 487-493.

[6] P.L. Lions, 'Approximation de points fixes de contractions', C.R. Acad. Sci. Paris, Sér. $A-B 284$ (1977), 1357-1359.

[7] S. Reich, 'Strong convergence theorems for resolvents of accretive operators in Banach spaces', J. Math. Anal. Appl. 75 (1980), 287-292.

[8] R. Wittmann, 'Approximation of fixed points of nonexpansive mappings', Arch. Math. 58 (1992), 486-491.

Departamento de Análisis

Universidad de Sevilla

Apdo. 1160

41080-Sevilla

Spain

e-mail: tomasd@us.es
Departamento de Análisis

Universidad de Sevilla

Apdo. 1160

41080-Sevilla

Spain

e-mail: glopez@us.es

Department of Mathematics

University of Durban-Westville

Private Bag X54001

Durban 4000

South Africa

e-mail: hkxu@pixie.udw.ac.za 